\title{
How ICT Changes the Landscape of Disaster Risk Management
}

\author{
Wei-Sen Li, Chi-Ling Chang, Ke-Hui Chen, and Yanling Lee \\ National Science and Technology Center for Disaster Reduction, New Taipei, Taiwan \\ li. weisen.ncdregmail.com
}

\begin{abstract}
Nowadays, ICT has been broadly applied to all trades and professions, because of instant end-to-end connectivity and at-hand convenience. For sake of better enhancing disaster resilience, scientists, engineers and practitioners are make the most use of information and communication technology to all phases of disaster risk management- reduction, preparedness, response and recovery. Especially at the IOT age, the powerful tools provide both macro- and micro- viewpoints in both physical and social vulnerabilities that could help to make decisions on disaster risk reduction or emergency response. How to massage all informative and multiple inputs to meet demands of different users that requires several elements to succeed: 1) To identify fields to apply ICT for; 2) To design information intelligence for action; 3 ) To disseminate information through various channels; 4) To build up public-private partnership in information sharing; 5) To carefully utilize social media. During last decade, though team work between emergency responders and scientists, Taiwan has developed a systematic approach to integrate scientific outputs with emergency operation amid times of typhoons. The results do prove well-organized information shared by ICT both increase efficacy of emergency operation and public awareness.
\end{abstract}

Keywords: Information communication technology (ICT), Disaster risk management, Emergency preparedness

\section{ICT offers backbones support to disaster risk management}

To fulfill end-to-end information facilitation, several key elements are required to build up a sound environment for decision support. These elements include 1) Data collection and monitoring system to raise situation awareness; 2) Broad-band wire-less telecommunications to gather and disseminate information; 3) Protocols and standards to form cross-platform application of information; 4) Spatial demonstration of disaster alerts and emergency to enhance risk understanding through systematic approach; 5) Adoption of artificial intelligence (AI) to analyze structured- and non-structured big data. With the five backbones, to be explained in the follow context, ICT plays an important role at disaster risk management and emergency preparedness [1].

Before, during and after a disaster, a network of sensors to collect and measure environmental changes is offering baselines to estimate possible degrees of im-pacts. For example, to decide threshold values of flood in a river basin, the design of a sensor 
network usually includes several gauges to measure rainfalls, water levels, soil moisture and operation of pump stations. Since lots of sensors could be installed in remote locations, well-established broad-band wireless telecommunications will help to transmit data for processing. Observations from recent large-scale disaster events, broad-band wireless telecommunication had proven to be the key of successfully-coordinated operations at affected sites. No matter search and rescue, emergency relief allocation, or shelter management needs location-based information, which must be in line telecommunication.

As more ICT devices are able to bring information or alerts to diverse end users, the general public, emergency responders or decision makers, international standardized protocols interoperate computer codes into functional machine commands to trigger actions. Such like the Public Waning System (PWS) for mobile phone, it is based on the Common Alerting Protocol (CAP) to deliver alter messages to and alarm end users. Following rapid development of mobile communication, recent protocol updates to PWS will further upgrade functions and capacity compared with previous version.

An effective message for individual user taking actions amid a developing disaster must contain geo-spatial location, time-dependent description and suggested actions. Usually, it is ideal to present an informative map with all factors mentioned above. Amid emergency operation for typhoon, commanding officials in emergency operation center (EOC) demand for a clear operation picture to make decision. The Fig. 1 is a sample for making decision on road closure. On the slide, it provides rainfall information (both hourly intensity and accumulation) and real-time images captured from closed circuit television cameras (CCTV) on a GIS map. The map offers clear and direct intelligence to ensure the countermeasure [2][3].

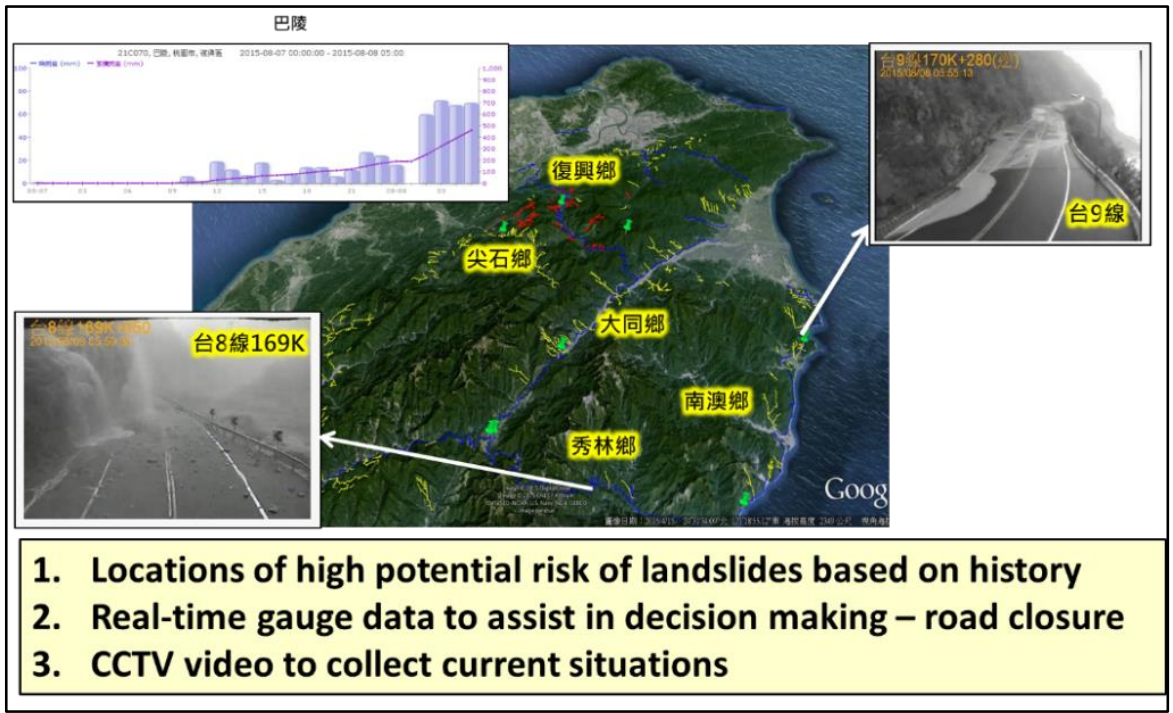

Fig. 1. Integration diverse information sources on a GIS map for decision support. 


\section{ICT applications for disaster risk reduction}

Direct benefits of ICT to disaster risk reduction are: 1) To connect early warning system with message deliver system for reaching out possibly affected areas and residents; 2) To assist in decision making through offering common operating picture for better inter- and intra- agencies coordination; 3) To create innovative channels for risk communication among all stakeholders; 4) To provide scenario-based impact assessment for emergency preparedness. In the past, prior to broad adoptions of ICT, it was too hard to deliver a comprehensive situation map for planning disaster risk reduction. Especially, when a large-scale disaster or cascading impacts occurred, the difficult and evolving situations could be very challenging to cope with.

Early warning system has been a focal issue since the South-East Asia Earthquake and Tsunami in 2004 [4]. From detecting earthquake, measuring tidal pressure change, relaying signals, to issuing warning, the whole process requires ICT's assistances to complete each procedure. One case in Taiwan, since 2013, a public warning system in operation is designed to send earthquake alter to $4 \mathrm{G}$ cellular phones by using Cell Broadcast Service (CBS). The service is a typical end-to-end system for alarming possibly affected areas through linkage between public and private sectors. Fig. 2 shows how relevant agencies join the mechanism to disseminate disaster alerts in seconds. In case of an earthquake, it might take 15-20 seconds to reach end users. The time differences depend on focal depth of hyper-center, user's distance to epicenter, user's location and signal strength of mobile phones.

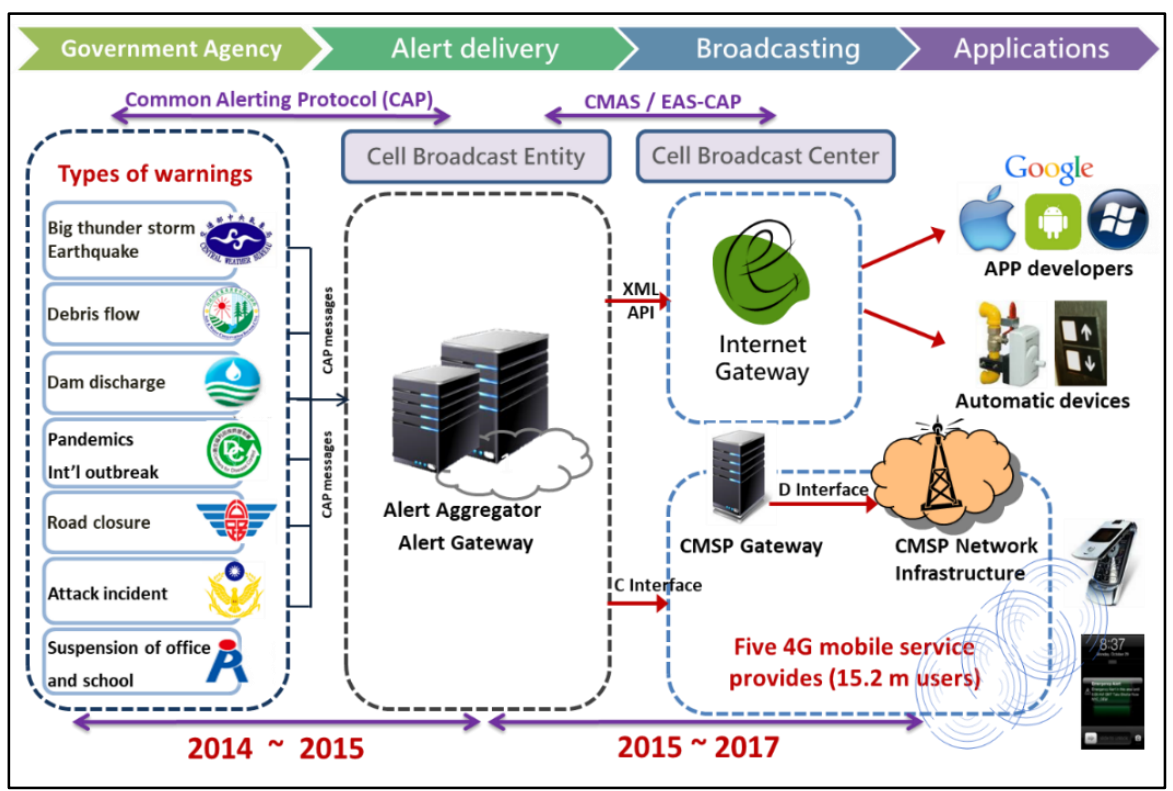

Fig. 2. The joint-effort mechanism to send alters through Cell Broadcast Service 
Web-GIS-based system is an ideal platform to present and share information when emergency responders are executing their missions at different spots. However, in case of no common platform existing, the overwhelming result could be too many systems to check at a time, and the situation might cause delays and gaps of information facilitation. In order to narrow down information gaps between central and local government, the National Science and Technology Center for Disaster Reduction (NCDR) has gradually set up a decision support system to integrate all existing data shared by individual agencies on it and offers user-oriented design to display situations. The system receives high appreciation of users for its easily unstainable information and flexibility to accommodate operation demands. The Fig. 3 is a layout of webpage to display geospatial information with situation briefing and real-time readings. The design ideas for the system is to keep information clean and neat on one page. At end user side, the system could overlap more GIS layers to tell local circumstances.

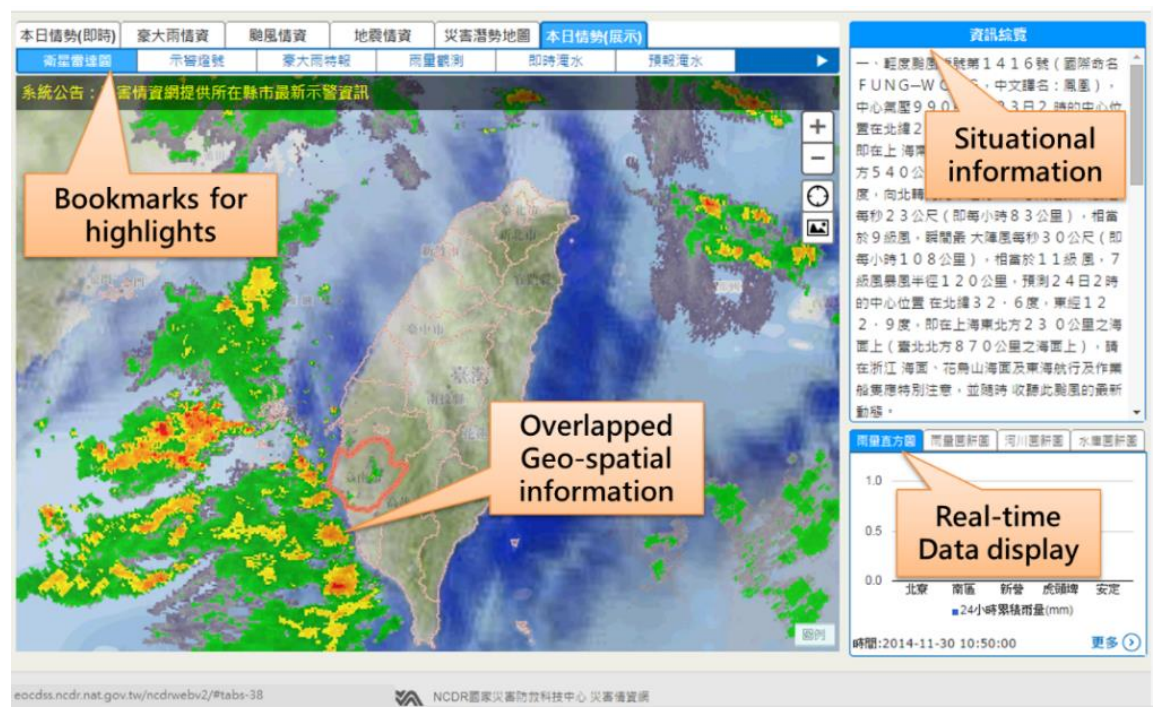

Fig. 3. A typical layout of "common operating picture" designed by NCDR

Public-private-partnership highlights engaging the whole society, especially business sector, to leverage resources for covering shortages that government can't immediately meet the demands. If any large-scale disaster hit, all residents must be eager for searching information on governmental websites to fetch the most updated news or notices. However, it is impossible for any agency to prepare huge Inter-net bandwidth for surging user at a very short period [5] [6]. An obvious phenome-non always repeated that most of governmental websites were down under too many simultaneous access requests. But Internet giants like Google, Yahoo or Amazon have always-ready and sufficient bandwidth to deal with access demand surges. A pilot project in 2013 brought NCDR and Google for collaboration in sharing disaster alerts and geo-spatial information on Google's platforms - Google Alert and Google Crisis Map. That is a creative 
leap in Taiwan to effectively outreach citizens to inform disaster alters through sharing open data with private sector. During implanting the project, NCDR also introduced CAP to relevant governmental agencies, which are the authorized ones to issue disaster alerts. The results from 2014, 2015 to 2016, shows millions of users receiving disaster alters through Google. That is a win-win combination to increase information coverage and fulfill social cooperate responsibility (CSR). Fig. 4 shows the numbers of end users receiving disaster alert or information from Google platform during 2014 - 2016.

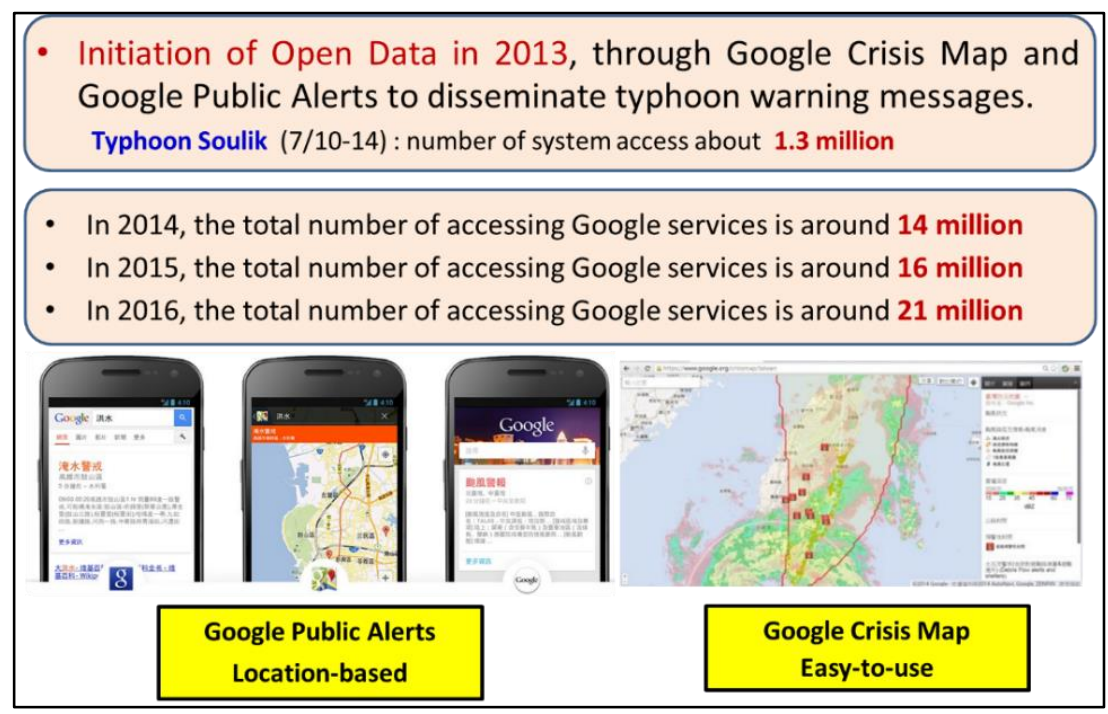

Fig. 4. Public-Private-Partnership with Google to distribute disaster alerts

\section{Practical improvements on disaster risk reduction}

Uncertainties at typhoon forecast do challenge decision making process at EOC, because unexpected casualties and losses might directly impact on commending officials. After years practices of collaboration between scientists and emergency responders in Taiwan, an innovative methodology has been developed and implemented that proves to be very effective for bridging scientific knowledge to emergency responders.

Because of focusing on impact assessment, all scientific outputs are transformed into understandable operation-oriented charts, GIS maps and suggestions on actions. During the transformation process, a learning-by-doing one, scientists and engineers co-work with emergency responders to catch what is the most appreciated information for them to make decisions and deliver operations. In case of typhoon emergency operation, at EOC, officers on duty eagerly want to know possible impacts brought by strong winds or torrential rains with clear information of "when?", "where?", "Scale of impact?" and "Scope of impact?" Through the process of impact assessment, scientists help to highlight possibly affected area with time-dependent factors (Fig. 5.). These information 
intelligences support pre-disaster operations like dispatching reinforced troops, conducting early evacuation or allocating equipment. The information designed for emergency responders never shows any equations or mentions about probabilistic equations. By doing in that way, all in-formation is clear and operational based on experiences and knowledge learned by extensive dialogues and opinion exchanges. In that way, a successful model of risk communication formulates a good eco-system, which proves "evidence-based" disaster risk management operable [7].

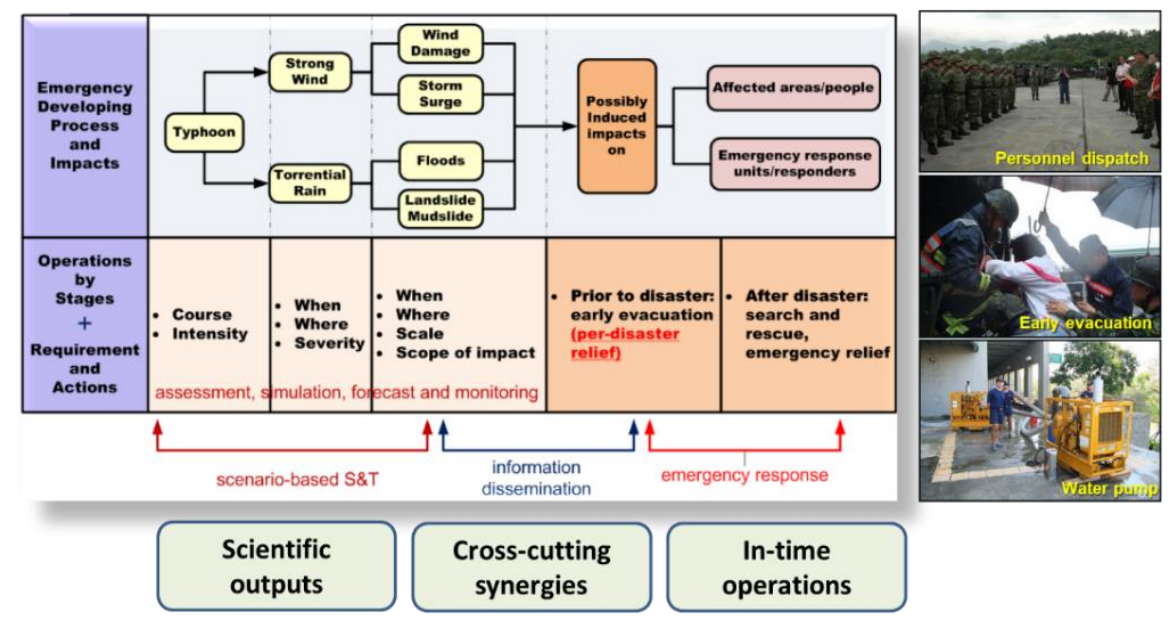

Fig. 5. Practical improvements on disaster risk reduction

\section{Conclusions}

Through broadly applying ICT for disaster risk management, the direct benefits include improving data collection; offering more dynamic and location-based in-formation display; creating direct channels to reach end users; shortening knowledge gaps between scientists and emergency responder; and enhancing quality of decision making. However, the process is still evolving at fast pace and all stakeholders should keep openminded to welcome future progress and implement changes through a team work.

\section{References}

1. Hsieh, S.H.: Achievements and Outputs of the Program on Applying Science and Technology for Disaster Reduction, 2011-2014, (2015).

2. . Tasy, C.Y: From Academic Research to Policy Making - A Case of Taiwan Effort in Disaster Reduction (2009).

3. Chen, L.C., Yen, C.L., Loh, C.H., Wei, L.Y., Lee W.C., Ho, H.Y.: Development and Implementation of Disaster Reduction Technology in Taiwan. Natural Hazards, 37 (1) (2006). 
4. Chen, L.C., Liu Y.C., Chan, K.C: Integrated Community-Based Disaster Management Program in Taiwan: A Case Study of Shang-An Village. Natural Hazards, 37 (1) (2006)

5. Yen, C.L, Loh, C.H., Chen, L.C., Wei, L.Y., Lee W.C., Ho, H.Y.: Development and Implementation of Disaster Reduction Technology in Taiwan. Natural Hazards, 37 (1) (2006)

6. Hsu, H.H, Chou, C., Wu, Y.C, Lu, M.M., Chen C.T., Chen, Y.M.: Climate Change in Taiwan: Scientific Report 2011 (Summary), National Science Council (2001).

7. Morakot Post-Disaster Reconstruction Council, Rebuilding a Sustainable Homeland with Innovation and United Efforts, Executive Yuan, Taiwan (2011). 